BULL. AUSTRAL. MATH. SOC.

$35 \mathrm{~K} 60,80 \mathrm{~A} 20$

VOL. $26(1982), 421-443$.

\title{
DIFFERENTIAL EQUATIONS AND THE REAL WORLD*
}

\author{
A.B. TAYLER
}

\begin{abstract}
As an illustration of the Oxford Study Groups with Industry project a number of applications of moving boundary problems for parabolic equations are described, and a possible model for the solidification of a binary alloy is discussed in detail. Further models of physical processes, which lead to non-linear and improper parabolic equations, are also presented in the hope of stimulating theoretical interest.
\end{abstract}

\section{Introduction}

The Oxford Study Groups with Industry for 14 years have been attempting to interact with British industry in the solution of scientific research problems which involve in some way or other the theory, application, or computation of differential equations. I shall not here describe the operation of the Study Groups, their impact on industry, nor their spin-off in terms of graduate education in Applied Mathematics in Oxford, see for example Tayler [27], [28], and Ockendon [16], but will attempt to demonstrate the benefits which such interaction may provide for the faculty member involved. Our experience is that good mathematics, and hence good research topics, often arise from real problems; and that by

Received 18 May 1982. The author should like to thank the Australian Mathematical Society for inviting him to their 1982 Applied Mathematics meeting and also the Indian Institute of Science, Bangalore for hosting him for three months during which time most of this paper was prepared.

* This paper is based on an invited lecture given at the Australian Mathematical Society Applied Mathematics Conference held in Bundanoon, February 7-11, 1982. Other papers delivered at this Conference appear in Volumes 25 and 26 . 
investigating current real problems an early start may be obtained on a topic capable of interesting mathematical development.

The difficulty in this approach lies in the variety of applications which real problems present, and the inevitable inadequacy of the applied mathematician's background knowledge of science. He or she must therefore be prepared to listen with humility to others with much less mathematical experience, to read in strange journals with irritating conventions, and to risk criticism in proposing simple mathematical models. A successful mathematical model usually evolves only after significant criticism of earlier models, and it is important that any model is given as much exposure as possible before extensive computation is applied or publication sought. It is therefore a tiring and time-consuming task to adopt this approach to a wide variety of real problems but the relevance of the problem is a powerful stimulus and the diversity a valuable education. These compensations might not however have been sufficient for the success of the Study Groups if it were not for the emergence of mathematical themes from diverse problems; themes which could be developed into novel mathematical ideas and methods, and provide conjectures for pure mathematicians to tackle.

To demonstrate a mathematical theme which has emerged from the Study Group Project I shall necessarily give a personal case-history which is inextricably mixed with contributions from other members of the Group. I have chosen to describe the area of moving boundary problems for parabolic equations; a topic which in the last ten years has acquired a substantial literature, for example Rubinstein [24], Ockendon and Hodgkins [18], Wilson, Solomon, and Boggs [30], and Elliott and Ockendon [7]. First in Section 2 a number of applications relevant to the theme will be briefly described and some results given, but without details. These applications are varied but the mathematical background is fairly well established. In Section 3 a particular problem, that of the solidification of an alloy, will be discussed for which the model is still controversial, but the problem is both relevant and important. Finally in Section 4 some mathematical extensions of the theme will be sketched, extensions which have not yet been fully justified by their application and which leave open a number of questions for rigorous analysis. 


\section{Phase change problems}

For the classical Stefan problem the temperature $u$, normalised so that $u=0$ is the phase change temperature, is related to the enthalpy $h(u)$ by the conservation law

$$
\frac{\partial h}{\partial t}=\beta \nabla^{2} u
$$

where $\beta$ is a constant diffusion coefficient (greater than 0 ). The non-dimensional enthalpy is defined by

$$
h=u, u<0 ; h=u+\lambda, u>0,
$$

where $\lambda$ is the non-dimensional constant latent heat (greater than 0 ). Thus if $u=0$ on a surface $F(x, y, z, t)=0$, there will be a discontinuity in $\nabla u$ across this surface which may be obtained from a physical heat balance. Alternatively a weak solution $u$ may be defined by an appropriate integral relationship involving a test function $\psi$, which reduces to ( 1 ) when $u$ is a classical solution, that is differentiable at least twice in space and once in time, and gives the appropriate jump conditious on $F=0$ in the form

$$
\lambda \frac{\partial F}{\partial t}=[\beta \nabla u]_{S}^{L} \cdot \nabla F,
$$

where suffices $S$ and $L$ indicate solid and liquid phases. The simplest problems occur with one space variable $x$ and phase-change boundary $x=s(t)$, so that $(3)$ reduces to

$$
x=s(t), u=0,-\lambda \dot{s}=\left[\beta \frac{\partial u}{\partial x}\right]_{S}^{L} .
$$

Oleinik [19] has shown that this weak solution exists, is unique, and the finite difference scheme

$$
h_{n+1}=h_{n}+B \delta t\left(\frac{\partial^{2} u}{\partial x^{2}}\right)_{n}, u_{n}=u\left(h_{n}\right),
$$

converges to it. This result forms the basis of the enthalpy method which solves (1) over a fixed domain by finite difference methods based on (5), necessarily obtaining the required weak solution with a phase change boundary satisfying (4). The result remains true in two of three space dimensions with an appropriate generalisation of (5). A number of 
applications require extensions of the classical problem.

\section{(a) Continuous casting}

In this process solidified metal is drawn with constant speed from a large molten reservoir which may be assumed to be at the solidification temperature $u=0$ because of mixing. If a thin sheet is formed by this process as in Figure 1 , then in a steady state ( 1 ) reduces to

$$
\frac{\partial u}{\partial z}=\beta\left(\frac{\partial^{2} u}{\partial y^{2}}+\frac{\partial^{2} u}{\partial z^{2}}\right), y<s(z)<1,
$$

with

$$
u=0, \lambda s^{\prime}(z)=\lambda\left(\frac{\partial u}{\partial y}-s^{\prime} \frac{\partial u}{\partial z}\right) \text { on } y=s(z)
$$

where the convection.term $\partial / \partial z$ has replaced the time derivative.
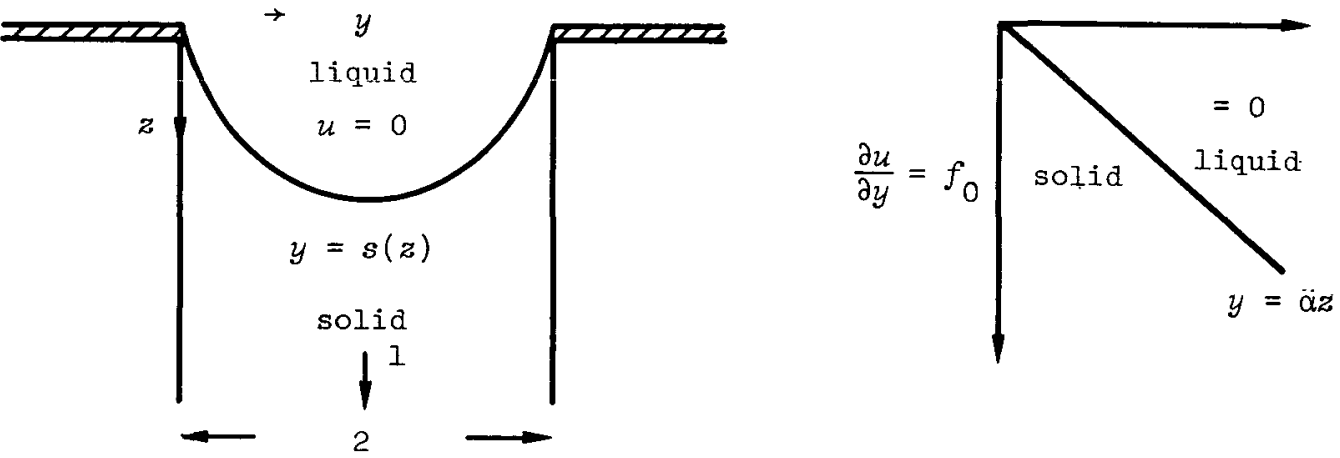

FIGURE 1a, b. Continuous casting

For a thin sheet we may neglect $z$ derivatives in comparison with $y$ derivatives to obtain a one dimensional stefan problem which can be solved by the enthalpy method on the fixed domain $0<y<1, z>0$. The approximation will not however be valid near the corners $z=0$, $y=0,2$, where $y$ and $z$ length scales are not necessarily of different orders of magnitude. A simple minded local expansion about $y=z=0$ with boundary condition $\partial u / \partial y+\mu u=f$ on $y=0$, gives $u \sim f_{0}(y-\alpha z)$ with $s \sim \alpha z$, where $\alpha$ is defined by

$$
\alpha^{2}-\frac{\lambda \alpha}{B f_{0}}+1=0
$$


This has no roots for $\lambda<2 \beta f_{0}$ and is not an acceptable asymptotic form; a possible form has been suggested by Blackwell and Ockendon [3] in which the phase change boundary does not pass through $y=z=0$.

(b) The shape of me1tpools

In laser welding, the laser beam and shield, assumed to be of circular cross-section, create a circular hot spot through a metal sheet which may be simply modelled as a region of constant temperature $u_{0}>0$ for constant heat flux on its boundary $\partial u / \partial r=-q_{0}<0$ ). By conduction a meltpool is formed round the laser beam and if the beam is moved the liquid metal will flow and convection effects must be included. A simple situation is the steady state formed when the metal is moving with constant speed relative to the laser as in Figure $2 a$. The heat conduction equation now becomes

$$
(v \cdot \nabla) u=\beta \nabla^{2} u,
$$

where $v=(0,0,1)$ in the solid, but has to be determined from flow equations in the liquid metal, with boundary conditions on the hot boundary $r=1$ and the phase change boundary $r=f(\theta)$. The stefan condition has the form ( 7 ) but in this case $u \neq 0$ in the liquid.

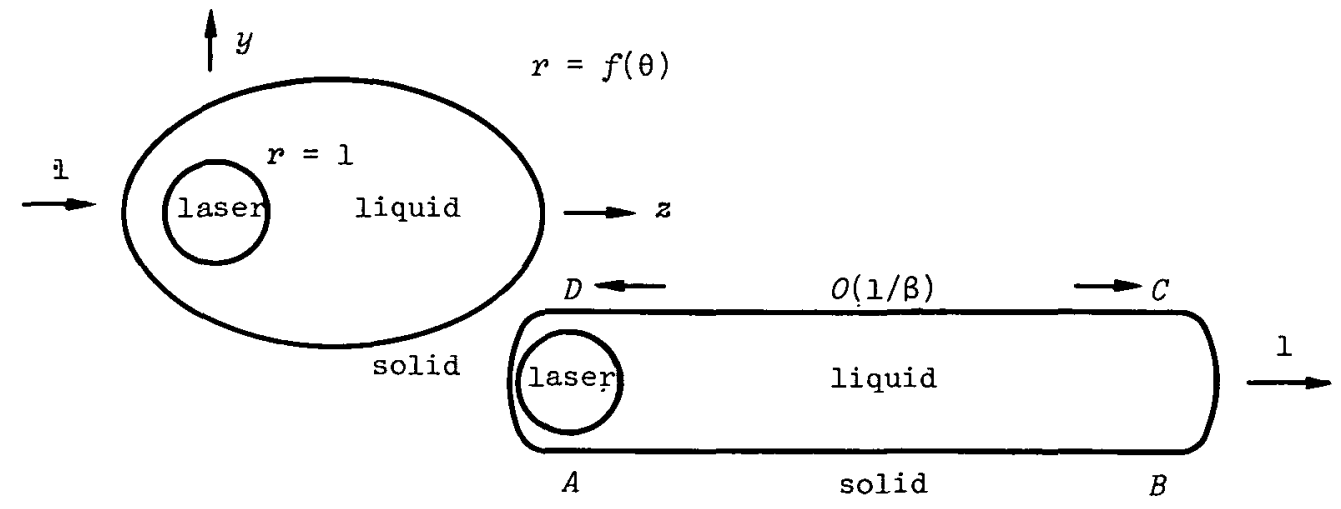

FIGURE 2a, b. Meltpools in laser welding $(B \sim 1$ and $B \ll 1)$

If conduction dominates convection ( $\beta \gg 1$ ) then the meltpool is roughly circular and has a large radius $R$ given by

$$
(2 \beta){ }^{\left(u_{0} /\left(1+u_{0}\right)\right)} \exp \left(\frac{-\gamma u_{0}}{1+u_{0}}\right) \text {, }
$$


where the temperature far from the laser is -1 and $\gamma$ is Euler's constant (see [23]).

When convection dominates conduction $(\beta \ll 1)$ then the meltpool is long and thin as in Figure $2 \mathrm{~b}$ and a variety of boundary layers occur adjacent to the phase change boundary. A total heat balance provides a relation between the length of the pool and the total heat flux out of the laser, and, if finite, implies that the pool length is $O(I / \beta)$. When $\beta$ is finite no results have been obtained, and stefan problems with convection in more than one space dimension pose difficult computational problems.

\section{(c) Resistance spot welding}

In this process an a.c. current is passed between two electrodes on either side of the two plates to be welded as in Figure $3 a$ and the shape of the meltpool, or nugget, is of interest. This meltpool is in the thickness of the metal sheets, unlike the previous problem where conditions were assumed to be uniform throughout the thickness, and for simplicity we consider only a one dimensional problem. The Joule heating creates a source density of heat so that

$$
\frac{\partial u}{\partial t}=\beta \frac{\partial^{2} u}{\partial x^{2}}+q
$$

where $q$ is assumed to be constant.
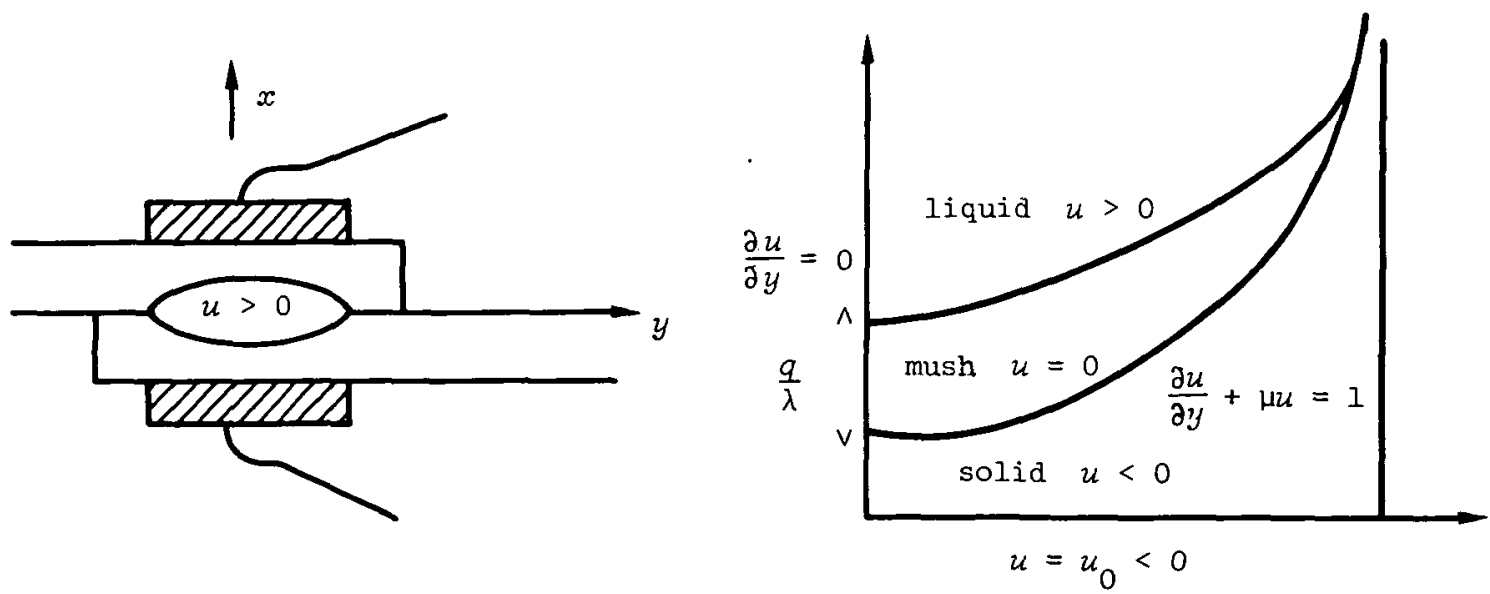

FIGURE 3a, b. Resistance spot welding 
The weak solution and enthalpy method has been extended to this problem by Atthey [2], and the solution obtained gives a region of constant temperature $u=0$, indicated in Figure $3 \mathrm{~b}$, in which the metal is neither solid nor liquid but is a 'mush'. Physical arguments may be used to confirm the existence of this mushy region and alternative models in which the solid is allowed to be superheated, that is can take on values $u>0$ without change of phase, lead to phase change boundaries which are unstable, as shown by Ockendon [17].

The non existence of a Stefan phase change boundary is a result which is perhaps a little unexpected.

(d) Vapourisation of a liquid in contact with a heated solid block

A layer of liquid is in contact with a heated block so that a region of vapour forms between the two. The liquid is pressurised to inhibit bubbles forming and we assume that the vapour layer is a one dimensional strip $0<x<s(t)$ as in Figure 4a, with a gas law $p(1+u)$ is constant, where $u=0$ is the phase change temperature and $u=-1$ is absolute zero.

$p=p_{0}$

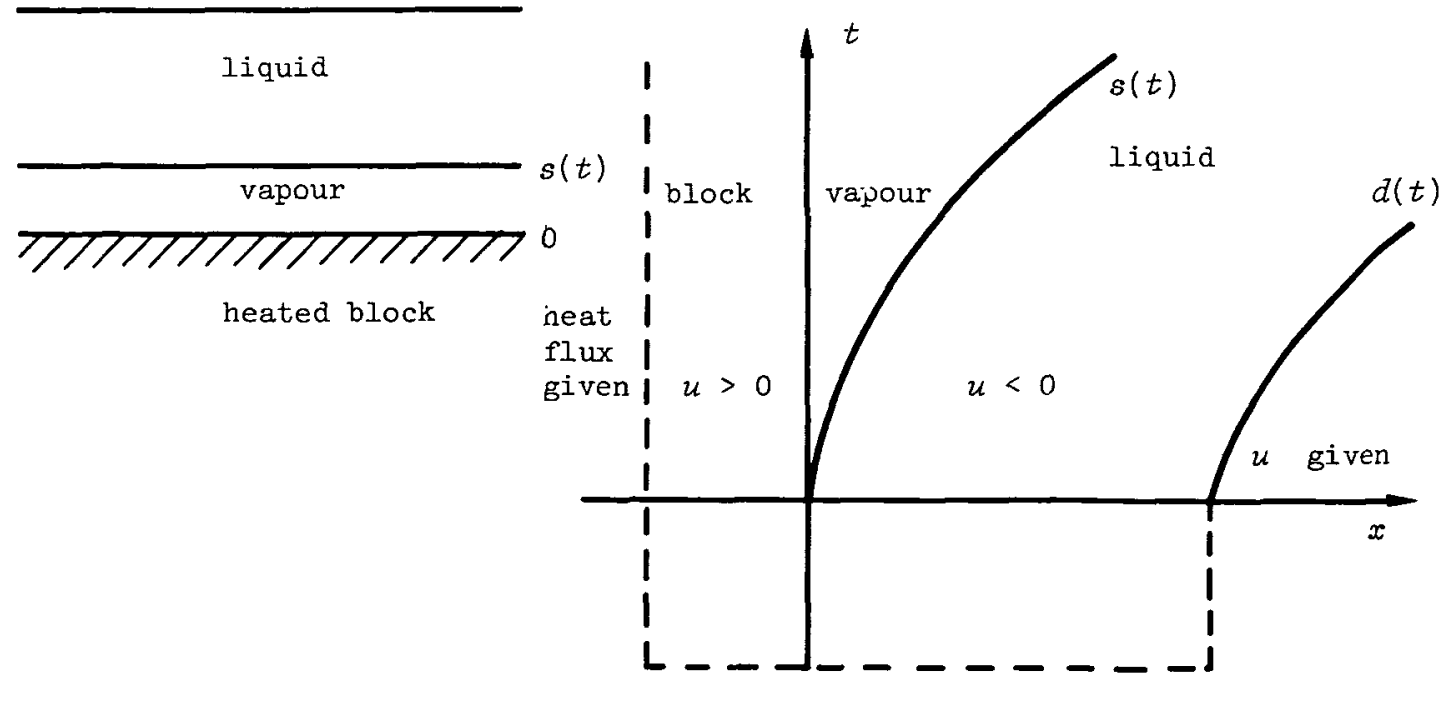

$u$ given

FIGURE $4 \mathrm{a}$, b. Creation of vapour phase 
Conservation of mass and energy give, on eliminating the density $\rho$,

$$
\frac{\partial u}{\partial t}+v \frac{\partial u}{\partial x}=(1+u) \frac{\partial v}{\partial x}=\varepsilon(1+u) \frac{\partial^{2} u}{\partial x^{2}}
$$

where $v$ is the gas velocity and $\varepsilon$ is a non-dimensional parameter possibly small. Small changes in the pressure are governed by a momentum equation but are of little interest. The vapour cannot be at rest because of the large change in densities between the two phases and from (10),

$$
v=\varepsilon\left(\frac{\partial u}{\partial x}-\left(\frac{\partial u}{\partial x}\right)_{x=0}\right) \text {. }
$$

The conditions at the phase change boundary are

$$
x=s(t), u=0,[\rho(v-\dot{s})]_{G}^{L}=0,
$$

together with a complicated expression for $[\beta(\partial u / \partial x)]_{G}^{L}$. However assuming that $\rho_{G} \ll \rho_{L}$ these conditions reduce to

$$
x=s(t), u=0, v_{L}=\dot{s}, \quad\left[\beta \frac{\partial u}{\partial x}\right]_{G}^{L}=\varepsilon \lambda\left(\dot{s}-v_{G}\right) .
$$

In the liquid, $s(t)<x<d(t)$, and from continuity $v=\dot{s}=\dot{d}$, so that

$$
\frac{\partial u}{\partial t}+\dot{s} \frac{\partial u}{\partial x}=\beta \frac{\partial^{2} u}{\partial x^{2}},
$$

and the various domains are shown in Figure 4b. A numerical attack on the problem using averaged forms of the equations (with respect to $x$ ) has been made (A. Booth, private communication), but difficulties arose for small values of $t$. A small time solution can however be obtained and gives that $s \sim t^{3 / 2}, v \sim t^{5 / 2}$, with a change in heat flux across the vapour of $O(t)$, provided $t \ll \varepsilon^{2 / 3}$ for by scaling $t$ with $\varepsilon^{2 / 3}$ an expansion for small $\varepsilon$ is obtained).

This provides the appropriate singularity for the creation of the new phase. However there is considerable doubt about the stability of the interface and no further computation has been performed. 
(e) Condensation of a binary mixture on a cooled surface

The change of phase of a mixture is determined by its equilibrium or eutectic diagram as shown in Figure $5 \mathrm{a}$, and the principal feature is that vapour of concentration $c_{G}$ condenses at temperature $u_{m}$ into liquid of concentration $c_{L}<c_{G}$ so that for mass conservation of the secondary material the vapour is enriched, thus lowering its phase change temperature. For small concentrations $c$ the relationships $c_{G}\left(u_{m}\right)$ and $c_{L}\left(u_{m}\right)$ may be taken as linear with $u_{m}=0$ for $c_{G}=c_{L}=0$. For each phase there will be a mass balance for $c$ which allows for molecular diffusion, an energy equation for the temperature $u$, and these equations are linked by conditions at the phase change boundary.
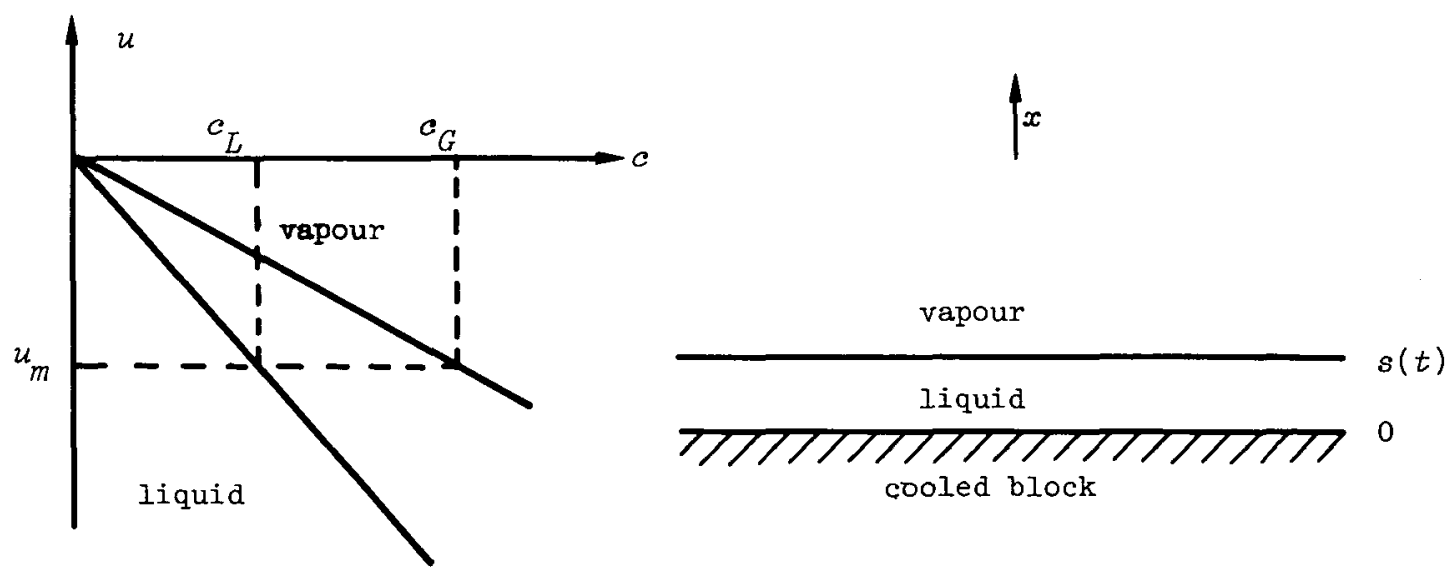

FIGURE 5a, b. Condensation of a binary vapour mixture

A simple example concerns a saturated vapour, concentration $c_{\infty}$, temperature $u_{\infty}$, such that $c_{G}\left(u_{\infty}\right)=c_{\infty}$, adjacent to a plane surface which is cooled for $t>0$ by imposing a temperature $u_{0}(t)<u$. The vapour condenses to form a liquid film of thickness $s(t)$ as in Figure $5 b$. For a thin film we neglect diffusion in the liquid so that $c=c_{L}\left(u_{m}\right)$, and assume that the heat flux through it is constant, equal to $-B\left(u_{m}-u_{0}\right) / s$. The displacement of the vapour by the liquid film is small so that in the vapour 


$$
\frac{\partial c}{\partial t}=D \frac{\partial^{2} c}{\partial x^{2}}, \frac{\partial u}{\partial t}=\beta \frac{\partial^{2} u}{\partial x^{2}},
$$

where $D$ is a molecular diffusion coefficient, and these are to be solved on $(0, \infty)$ in this approximation. Given $c$ (in terms of $u_{m}$ ) on $x=0$, and $c=c_{\infty}$ for $t=0$ and $x+\infty$, (13) may be solved to give $(\partial c / \partial x)_{x=0}$ in terms of $u_{m}$. The heat conduction equation may also be solved to give $(\partial u / \partial x)_{x=0}$ in terms of $u_{m}$. These expressions are then substituted in the jump conditions on the phase change boundary, namely

$$
\left[\beta \frac{\partial u}{\partial x}\right]_{L}^{G}=-\lambda \dot{s},\left[D \frac{\partial c}{\partial x}\right]_{L}^{G}=-\dot{s}[c]_{L}^{G},
$$

so that two relations may be obtained between $s, \dot{s}$ and $u_{m} \cdot \mathrm{A}$ complicated integro-differential equation for $s$ results, which for small $t$ implies that $s \sim t^{3 / 2}$ and $u_{m}-u_{0} \sim t^{2}$.

An extension of the enthalpy method to deal with weak solutions of pairs of diffusion equations defined by (13) and (14) has been developed and used in the problem of the solidification of an alloy, which we discuss in more detail in the next section.

\section{Solidification of binary alloy}

Consider a molten binary mixture being cooled in a one dimensional mould, $0<x<2$, by heat flow through the ends of the mould. The concentration $c$ of secondary material will have an equilibrium diagram of the same general form as that of the saturated vapour in problem 2 (e) above, so that the problem is to solve equations (13) with jump conditions (14) at the phase change boundary. For the alloy we can reasonably assume that the liquid and solid densities are the same so that there is no flow and do not have to restrict the problem to a thin solidified layer. A typical concentration profile, as shown in Figure $6 a$, describes the solution, see for example Flynn [8], and demonstrates the discontinuous enrichment at the phase change boundary and the diffusion into the liquid. The corresponding temperature profile will be monotonic increasing in $0<x<1$ with a slope discontinuity at $x=s$, so that the concentration profile has the same general form as a function of $u$ as of $x$. This 
profile can be superimposed on the equilibrium diagram and is shown as a dashed line in Figure $6 \mathrm{~b}$, giving a description of the material state at time $t$. There is a clearly defined phase change boundary at $x=s(t)$, $u=u_{m}(t)$, provided this dashed line does not intersect the region between the liquidus and solidus boundaries defined by $c+k_{L} u=0$ and $c+k_{s} u=0, k_{s}<k_{L}$. A situation in which the state lies between the liquidus and solidus boundaries is said to be constitutionally supercooled.
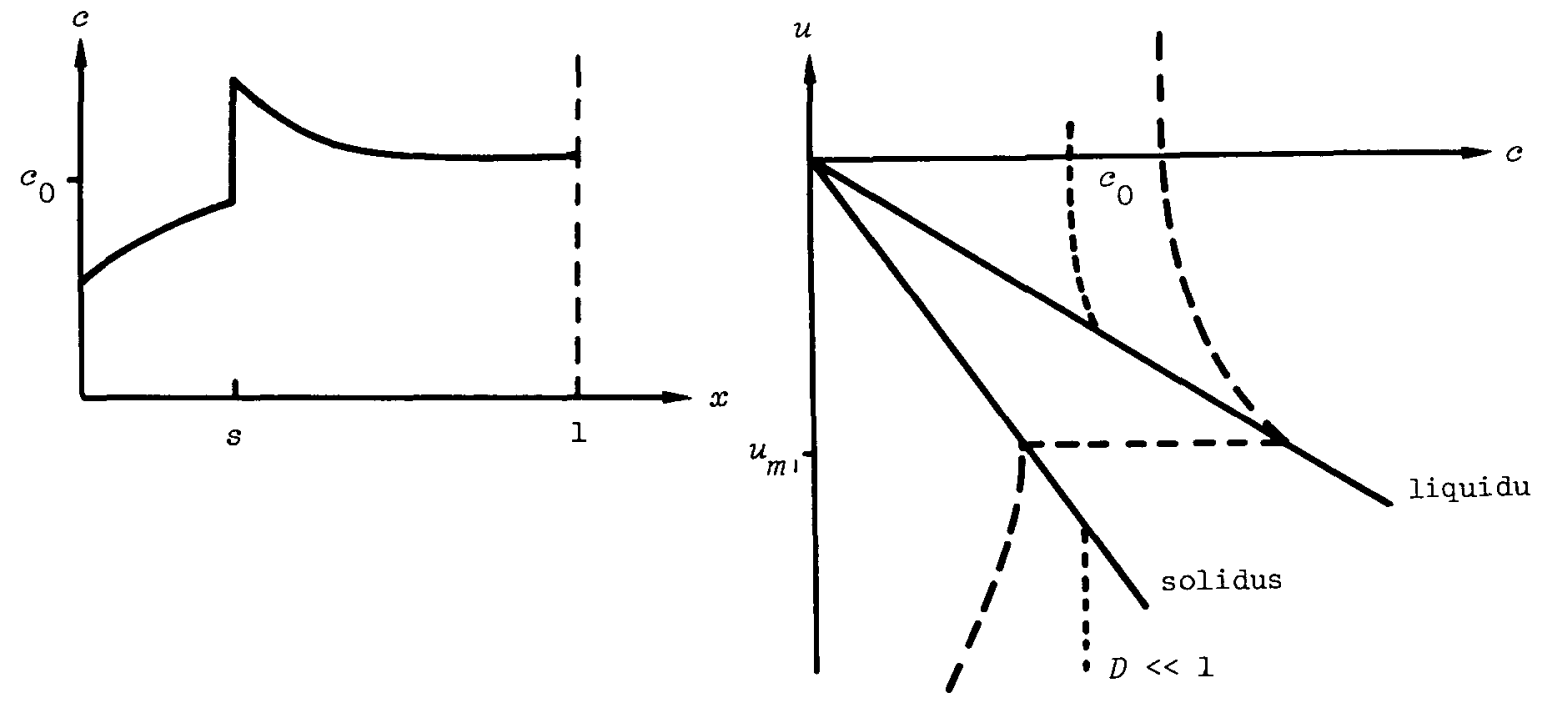

FIGURE 6a, b. Solidification of a binary alloy

One common practical situation is that the non-dimensional diffusion coefficient $D$ is much less than $B$, and scaling so that $B=1$, $D \ll 1$. An asymptotic solution of (13) and (14) is then $c=c_{0}$, $x<s(t)$, and for $x>s(t)$,

$$
c=c_{0}\left(1+\left(\frac{k_{L}}{k_{s}}-1\right) e^{-\dot{\boldsymbol{\delta}}(x-s) / D}\right)+O(D),
$$

where we have assumed that the diffusion coefficient in the solid is zero. This implies that $\partial c / \partial x \sim \dot{s} / D$, and, from (14), $\lambda \dot{s} \sim \partial u / \partial x$, so that $|c c / d u|$ (at constant $t$ ) will be large, and constitutional supercooling cannot be avoided for finite $\lambda$.

The occurrence of constitutional supercooling corresponds to the 
existence of the Mullins-Sekerka instability in the phase change boundary, see Ockendon [17], and it seems likely that, as in example 2 (c) but for a slightly different reason, the phase change boundary is replaced by a region whose thickness may not be small. This region is analogous to the mushy region of problem 2 (c) but the temperature in it is not constant. Outside this region the material state must either be liquid or solid, and if $D \ll<$ only small changes in concentration can occur across it so that there have to be $O(1)$ changes in temperature, as shown by the dotted line in Figure 6b. This implies that the thickness of the region is $O(1)$ and a macroscopic model for it is needed.

Before attempting to construct such a model it is interesting to examine a possible extension of the enthalpy method to equations (13) and jump conditions (14) proposed by Crowley and Ockendon [6]. By defining chemical activity $a$, such that $c=k_{L} a$ in the liquid, and $c=k_{s} a$ in the solid, then both $u$ and $a$ are continuous across the phase change boundary $u+a=0$, and the following numerical procedure is proposed:

$$
\begin{aligned}
h_{n+1} & =h_{n}+\delta t \beta\left(\frac{\partial^{2} u}{\partial x^{2}}\right)_{n} ; c_{n+1}=c_{n}+\delta t \bar{D}\left(\frac{\partial^{2} a}{\partial x^{2}}\right)_{n} ; \\
u_{n} & =u\left(h_{n}, c_{n}\right), a_{n}=\alpha\left(h_{n}, c_{n}\right) ;
\end{aligned}
$$

and $\bar{D}$ has the value $k_{8} D$ or $k_{L} D$ depending on the phase. However there is no convergence result available for this procedure, nor indeed can the weak solution be defined; also difficulties arise because the transformation $(h, c) \rightarrow(u, a)$ is not defined in the region $h+c / k_{s}>0$ and $h+c / k_{L}<\lambda$. Two ways of overcoming this latter difficulty are suggested, which are essentially equivalent although based on different arguments. The more physical argument considers a volume fraction of the material mixture to be solid and assumes that $c$ and $h$ are linear in $f$ so that

$$
c=c_{s} f+c_{L}(1-f)=-u\left[k_{s} f+(1-f) k_{L}\right] \text {, }
$$

and

$$
h=u+\lambda(1-f) \text {, }
$$


Eliminating $f$ an equation for $u$ in terms of $c$ and $h$ is obtained which has an appropriate real root in the region where the transformation $(h, c) \rightarrow(u, a)$ is not defined. In addition $u+a=0$ in this region so that $a$ is also determined. The method has been successfully applied when $D \sim O(1)$, giving good agreement with similarity solutions which exhibit no mushy region. For smaller values of $D$ the modification (17) and (18) has to be used in a finite region of $x$ which is then interpreted as a mushy region. In some averaged sense, therefore, the equations (13), (17) and (18) are being used as a model in this region.

The experimental evidence suggests that dendrites grow on the advancing solid phase, so that a more physical description of this region between the solidus and liquidus would take account of this three dimensional structure. In practice the dendrites have irregular crosssection and may grow branches, but a simple model considers the dendrites as smooth slowly tapering cylinders for most of their length as in Figure 7a, recognising that there will inevitably be end corrections necessary at the solidus $x=s_{1}(t)$ and liquidus $x=s_{2}(t)$ (the base and tip respectively). (This totally neglects the initial stages of growth of a dendrite and a challenging problem is to attempt a demonstration of the growth of an instability into a dendrite.)

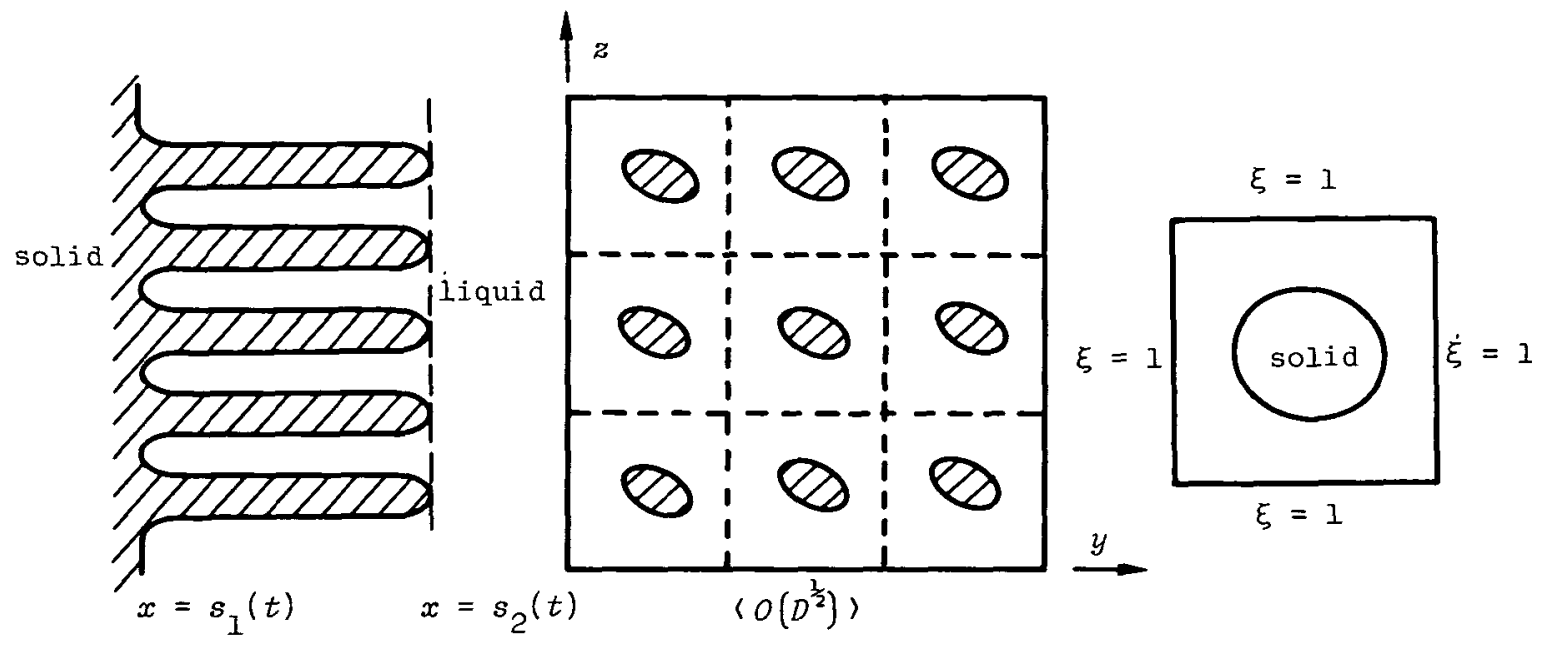

FIGURE $7 a, b$. Model for region of dendritic growth 
Then a unit cross-section of the material may be considered as a regular array of growing cells as in Figure $7 \mathrm{~b}$. The number of cells per unit area is not known but is unimportant for our purpose, however we assert that the cell size is $O\left(D^{1 / 2}\right)$, the lateral diffusion scale. An average temperature $u(x, t)$ and fraction solid $f(x, t)$ may be defined, together with some appropriate growth variable $\xi$ in the $(y, z)$ plane so that $0<\xi<1$, and $\xi=f$ is the boundary of each dendrite cross-section. If we also assume that diffusion in the $x$-direction (normal to the crosssection) is unimportant and that there is no diffusion in the solid, a simple model for the diffusion of concentration $\tilde{c}(\xi, t)$ in the crosssection is

$$
\frac{\partial \tilde{c}}{\partial t}=\frac{\partial^{2} \tilde{c}}{\partial \xi^{2}}, f \leq \xi \leq 1,
$$

where on $\xi=f$,

$$
\tilde{c}=-k_{L} u, \frac{\partial c}{\partial \xi}=\left[k_{L}-k_{g}\right] u \dot{f} .
$$

Also on $\xi=1, \partial \tilde{c} / \partial \xi=0$; and $t$ is measured from $t_{0}$ where $x=s_{2}\left(t_{0}\right)$ and $c=c_{L}\left(t_{0}\right)$ at $t=t_{0}$. Since however $D \ll 1$, $c_{L}=c_{0}+O(D)$ and, if $u$ were given, (19) and (20) define a single phase Stefan problem with variable 'latent heat', capable of solution by numerical methods. Since $u$ is not known this provides a functional dependence of $f$ on $u$ which we denote by

$$
f=F_{t}(u) \text {. }
$$

Note that it is the time variation of $u$ which is significant, $x$ only appears as a parameter in the problem. In solving this problem the dendrite boundary may still be unstable and this will depend on the rate at which $u$ decreases in time. If this is too fast then subsidiary dendrites or branches will grow from the main dendrites, and difficulties are likely to occur in the computation. It is however possible for the dendrites to be stable now that the diffusion length scale is of the same order as the length scale of the moving front.

Thus in this model the concentration diffusion equation (13) is abandoned together with (17) and replaced by (21), retaining (18) and the 
heat conduction equation in enthalpy form. This may be simplified to

$$
\begin{array}{rlrl}
\frac{\partial u}{\partial t}-\beta \frac{\partial^{2} u}{\partial x^{2}} & =\lambda \frac{\partial}{\partial t} F_{t}(u), \quad s_{1}<x<s_{2}, \\
& =0, & & 0<x<s_{1}, s_{2}<x<1,
\end{array}
$$

together with conditions that $u$ and $\partial u / \partial x$ are continuous on $x=s_{1}$ and $s_{2}$. Moreover at $x=s_{1}, u=-c_{0} / k_{s}+O(D)$ and at $x=s_{2}$, $u=-c_{0} / k_{L}+O(D)$, together with the usual boundary conditions on $x=0$ and 1 and $t=0$.

This model may be formulated as a version of the enthalpy method but numerical results are not yet available. The average concentration will be almost constant but the concentration distribution in the solidified crosssection is to be obtained in each cell from $\tilde{c}=-k_{s} u(x, t)$, evaluated on $\xi=f(x, t)$ by eliminating $t$. This is necessarily incomplete data since some assumption about the spacing of the dendrites and their crosssectional shape is needed to complete the picture and make comparison with experimental data possible. For values of $D$ which are not very small, but small enough for an $O(1)$ dendritic region to form, the model (19), (20), (22) should still be relevant but there will be a complicated interaction between (19) and (22) since the values of $u$ at $x=s$, and $s_{2}$ will depend on $s_{1}$ and $s_{2}$.

A similar model may be proposed for the steady continuous casting of an alloy, a process described in problem 2 (a). The significant difference is that the experimental evidence indicates that three dimensional grains grow rather than dendrites, and solidification occurs. An argument for this is that the turbulent liquid motion prevents dendrites forming and nucleation sites occur on which solid particles grow. Equations (19) and (20) may still be used as a model if now $\xi$ is a three dimensional growth variable for a moving grain on the length scale $D^{1 / 2}$. This gives an identical problem to (2l) above if $z$ is appropriately identified with $t$. 


\section{Other generalisations of the diffusion equation}

In Section 2 a number of applications of the more or less established theory of parabolic equations were described, and in Section 3 a particular problem, for which the mathematical model is still not agreed, was

discussed. Such a model in final form, will create a need for further pure theory about pairs of linked parabolic equations and possibly provide the basis for useful conjectures. It is interesting to examine some other models for real problems which have stinulated extensions to the theory of parabolic equations and further conjectures.

(a) Non-linear diffusion coefficients

The equation

$$
\frac{\partial u}{\partial t}=\frac{\partial}{\partial x}\left(u^{n} \frac{\partial u}{\partial x}\right)
$$

is a useful model for describing a number of physical situations, for example :

(i) the profile of a thin highly viscous oil film spreading under gravity on a horizontal surface (Buckmaster [5]),

(ii) the infiltration of moisture into a dry soil (Philip [21]),

(iii) the percolation of a gas through a porous medium (Muskat [15]),

and many others including the Von Mises transformation for flow in a boundary layer on a semi-infinite flat plate. In case (i), $n=3$, case (ii), $n$ is empirical and both 5 and 6 have been suggested, case (iii) for an isothermal gas $n=1$ and for an isentropic gas $n=\gamma$, the ratio of the specific heats.

For equation (23) Oleinik et al [20] have shown that the classical maximum/minimum theorem for the case $n=0$ may be replaced by a comparison theorem. This states that if $u_{1}$ and $u_{2}$ are both solutions of (23) and $0 ₹ u_{1} ₹ u_{2}$ for all $x$ at $t=0$, then $0 ₹ u_{1} ₹ u_{2}$ for all $t>0$ for which they both exist. Kalashnikov [10] has extended this to weak solutions, which are unique if defined appropriately, and there is particular interest in solutions with compact support, that is bounded by a free boundary beyond which $u \equiv 0$. To obtain the conditions holding on 
such a free boundary $x=s(t)$ needs careful analysis, but a simple demonstration is to integrate (23) over some suitable domain $D$ and convert it to the line integral

$$
\int_{\partial D} u^{n} \frac{\partial u}{\partial x} d t+\int_{\partial D} u d x=0 .
$$

With this as a starting point for non-classical solutions, possible discontinuities are given by

$$
\left[u^{n} \frac{\partial u}{\partial x}\right]_{-}^{+}=-\dot{s}[u]_{-}^{+}
$$

Thus, in some appropriate limiting sense, the required free boundary, adjacent to a region $u \equiv 0$, satisfies

$$
x=s, \frac{\partial}{\partial x}\left(u^{n}\right)=-n \dot{s}, u=0 \text {. }
$$

This clearly demonstrates the 'infinite speed' of the free boundary for the linear diffusion equation when $n=0$, but allows a finite 'wave speed' for all other values of $n$, with finite profile slope in the case $n=1$.

Two further interesting properties of (23) may be demonstrated by the exact solution due to Knerr [13],

$$
\begin{aligned}
u & =\frac{(-x)^{2 / n}}{(2+(4 / n))^{1 / n}\left(t_{0}-t\right)^{1 / n}}, x<0, \\
& =0, x \geq 0, \text { for } 0<t<t_{0} .
\end{aligned}
$$

This has a stationary free boundary $x=0$ for a finite time $t_{0}$, which depends on the initial data at $t=0$, and blows up at $t=t_{0}$. Other solutions have been constructed by similarity methods by Lacey et al [14] which have a free boundary which waits for a finite time $t_{0}$ and then moves off satisfying (24) above. In fact the wealth of similarity solutions is remarkable and they may be used with the comparison theorem to make statements and conjectures about the general initial value problem. One such result due to Aronson et $a l[1]$ is that a free boundary at $x=0$ at time $t=0$ will wait if locally the profile slope is less than or equal to $o\left(x^{2 / n}\right)$ but will have a finite waiting time. Kamin [11] proves 
that for large time a class of initial value problems will approach similarity solutions. A perturbation approach for $n$ small by Kath and Cohen [12] examines the solution of (23) near $t=t_{0}$ and relates it to solutions of $\partial v / \partial t=(\partial v / \partial x)^{2}$ which exhibit shocks, with the time for shock formation related to the waiting time.

(b) Diffusion coefficients which change sign

Much of existing theory of parabolic equations is for strictly positive diffusion coefficients even in the linear case, but applications arise in which the coefficient has a zero on a prescribed curve in the $x$, $t$ plane (called for convenience a transition curve) or on an unknown curve in the non-linear case. Methods of solution appear to depend on the type of transition curve, which may therefore play a role in any development of existence and uniqueness theorems. The simplest example arises from the heat transfer problem with small conductivity in which a semiinfinite flat plate in a prescribed flow is instantaneously heated at time $t=0$, where it is assumed that the flow is unchanged by the heat transfer. The temperature $\theta(x, t)$ then satisfies

$$
\frac{\partial \theta}{\partial t}+u \frac{\partial \theta}{\partial x}+v \frac{\partial \theta}{\partial y}=\frac{\partial^{2} \theta}{\partial y^{2}},
$$

where $(u, v)$ is the prescribed flow and $\partial^{2} \theta / \partial x^{2}$ has been neglected in comparison with $\partial^{2} \theta / \partial y^{2}$.

For an inviscid flow $u=1, v=0$ and the problem is self similar in variables $z=v / 2 \sqrt{t}, \tau=t / x$, reducing to

$$
\frac{\partial^{2} \theta}{\partial z^{2}}+2 z \frac{\partial \theta}{\partial z}=4 \tau(1-\tau) \frac{\partial \theta}{\partial \tau},
$$

with boundary conditions $z=0, \theta=1, z+\infty, \theta+0 ; \tau=0$, $\theta=0$ and presumably $\tau \rightarrow \infty, \theta \sim \operatorname{erfc} z$. That is we expect a transition from a steady leading edge layer better described in a variable $\eta=y / 2 \sqrt{x}=a \sqrt{\tau}$ to an unsteady layer for an infinite flat plate. This problem has an exact solution with different functional forms in $0<\tau<1$ and $1<\tau<\infty$, although on $\tau=1, \partial \theta / \partial \tau$ is not continuous. The solution is obtained by solving forwards in $\tau$ from $\tau=0$ and backwards 
in $\tau$ from $\tau \rightarrow \infty$, thus requiring boundary data to be given at both $\tau=0$ and $\tau \rightarrow \infty$. The two forms obtained fit together because the transition line is the characteristic $\tau=1$. It is interesting to note that Stewartson [25] used the same equation as a simple linearised model for the transition from the leading edge Blasius solution to the far down stream Rayleigh solution in the problem of an impulsively moved semiinfinite flat plate in a viscous fluid. The fully non-linear problem when the transition line is not a characteristic was discussed 22 years later by him in [26].

A more complicated example is discussed by Riley [22] when the flow is that of a viscous boundary layer so that $u$ and $v$ are defined in terms of the Blasius function $f(n)$. In this case the transition line is defined by $\tau f^{\prime}(n)=1$ and is sketched in Figure 8a. A simpler case discussed by Tayler and Nicholas [29] is that for slow flow with a constant pressure gradient when $u=y, v=0$ and the appropriate similarity variable is $\tau=t^{3 / 2} / x$ with leading edge variable $\zeta=y / x^{1 / 3}=2 z \tau^{1 / 3}$. In this case (25) reduces to

$$
\frac{\partial^{2} \theta}{\partial z^{2}}+2 z \frac{\partial \theta}{\partial z}=6 \tau(1-z \tau) \frac{\partial \theta}{\partial \tau},
$$

with a transition line $z \tau=1$, also shown in Figure 8 a.
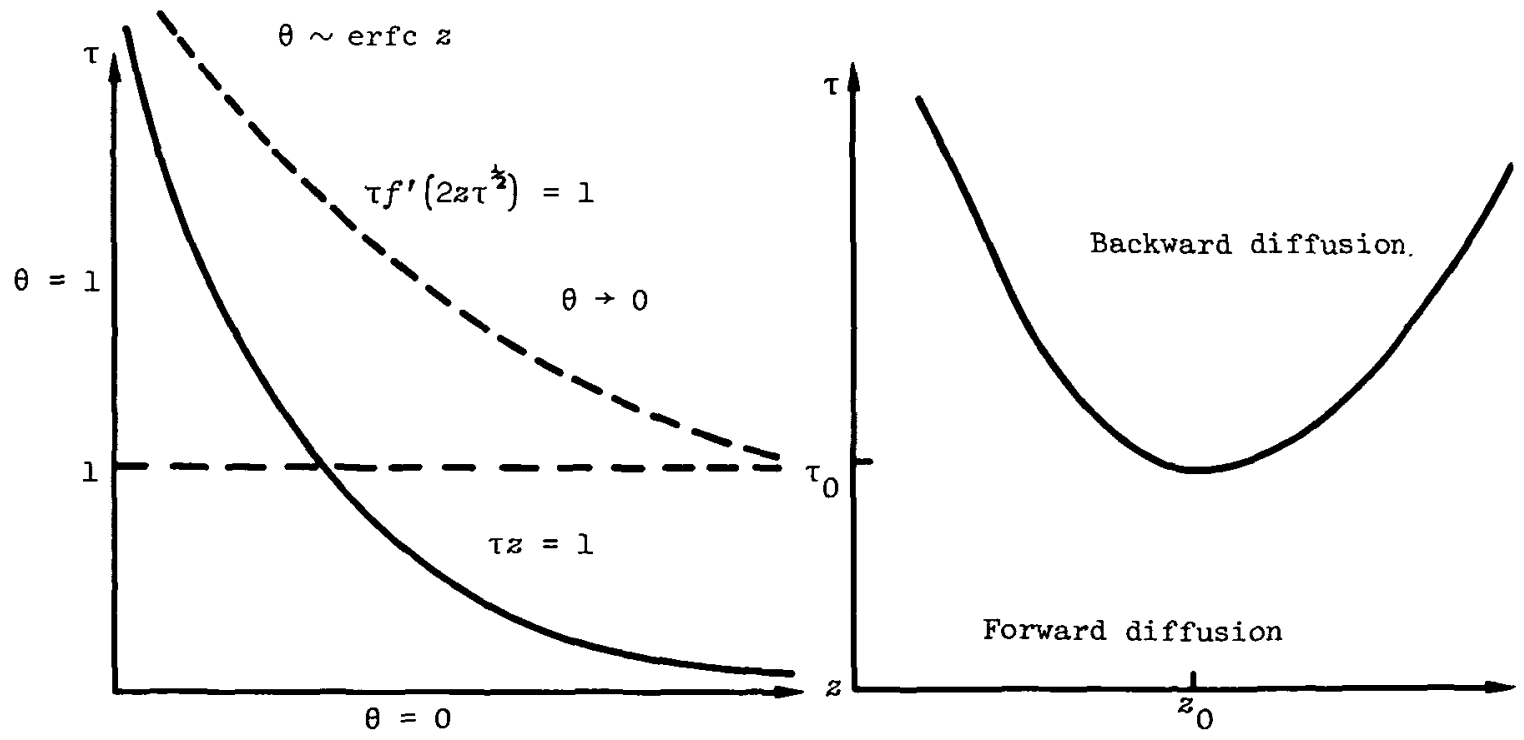

FIGURE $8 a$, b. Transition curves for improper diffusion equations 
A well posed problem is presumably $\theta$ given around the quadrant as before (omitting $0<z<1 / \tau$ as $\tau \rightarrow \infty$ ), and with such data sensible numerical results, reviewed by Ingham [9], have been obtained for both problems. However in both these cases the transition line meets each characteristic at most once, and a third type of problem occurs when the transition line has a turning point. An example discussed by Brown and Riley [4] is the problem of flow due to convection past a suddenly heated semi-infinite vertical flat place. The transition curve now has a minimum at $\left(z_{0}, \tau_{0}\right)$ as in Figure $8 \mathrm{~b}$, and all numerical methods applied so far give rise to serious errors in the neighbourhood of this point. One may speculate that the reason for this is the existence of an essential singularity at $\left(z_{0}, \tau_{0}\right)$ which is not amenable to finite difference techniques.

\section{Closing remarks}

Other themes such as homogenisation, non-Cauchy data for hyperbolic systems, and thin layer procedures, have emerged from the Study Group problems but the occurrence of parabolic equations in some form has dominated other types. At the last count in 1980 , out of 61 problems with which we made some useful progress, 34 were concerned with parabolic, 13 with elliptic, 10 with hyperbolic, and 4 with ordinary differential equations in some loose classification. Moreover 28 involved free or moving boundaries, although not all of these were of parabolic type. This dominance is not reflected in the emphasis given to parabolic equations in university courses and may account for the fact that so many parabolic problems were brought to us for help.

A more general implication for university education in applied mathematics is the desirability of giving students experience of modelling non-standard problems, since this is what they will be expected to do if they wish to apply their skills in industry. The importance of using a simple model as an aid to thinking about a problem, even if the model is inadequate in several respects, must be emphasised and the discussion of such models in small groups can be very rewarding.

Finally a very personal view is that applied mathematicians should not be afraid to distinguish themselves from pure mathematicians, and from 
engineers and physicists. Theorem and proof are not the only means to progress in mathematics; and breadth and diversity of mathematical application may give invaluable analogy. This view if accepted has serious implications for education in Applied Mathematics which fortunately it would be inappropriate to develop here.

\section{References}

[1] D.G. Aronson, L.A. Caffarelll and S. Kamin, "How an initially stationary interface begins to move in porous medium flow", Preprint.

[2] D.R. Atthey, "A finite difference scheme for melting problems", $J$. Inst. Math. App 1.13 (1974), 353-366.

[3] J.H. Blackwell and J.R. Ockendon, "Exact solution of a Stefan problem relevant to continuous casting", Int. J. Heat Mass Transfer (to appear).

[4] S.N. Brown and N. Ri ley, "Flow past a suddenly heated vertical plate", J. Fluid Mech. 59 (1973), 225-237.

[5] J. Buckmaster, "Viscous sheets advancing over dry beds", J. Fluid Mech. 81 (1977), 735-756.

[6] A.B. Crowley and J.R. Ockendon, "On the numerical solution of an alloy solidification problem", Int. J. Heat Mass Transfer 22 (1979), 941-947.

[7] C.M. Elliott and J.R. Ockendon, Weak and variational methods for free and moving boundary problems (Pitmans, London, 1981).

[8] C.P. Flynn, Fundamentals of metal casting (Addison-Wesley, Reading, Massachusetts; Palo Alto; London; 1963).

[9] D.B. Ingham, "Singular parabolic partial-differential equations that arise in impulsive motion problems", J. Appl. Mech. 44 (1977), 396-400. 
[10] A.C. Калапников [A.S. Kalašinkov], "Задача Коши в классе растуших фунцсй дтя уравнений типа нестагионарной фитырации" [The Cauchy problem for equations of non-stationary seepage type], Vestnik Moskov. Univ. Ser. I Mat. Meh. 6 (1963), 17-27.

[11] S. Kamin, "Similar solutions and the asymptotics of filtration equations", Arch. Rational Mech. Anal. 60 (1976), 171-183.

[12] W.L. Kath and D.S. Cohen, "Waiting time behaviour in a nonlinear diffusion equation", Stud. Appl. Math. (to appear).

[13] Barry F. Knerr, "The porous medium equation in one dimension", Trans. Amer. Math. Soc. 234 (1977), 381-415.

[14] A. Lacey, J.R. Ockendon and A.B. Tayler, "Waiting time solutions of a non-linear diffusion equation", SIAM J. App Z. Math. (to appear).

[15] M. Muskat, The flow of homogeneous fluids through porous media (McGraw Hill, New York, London, 1937).

[16] J.R. Ockendon, "Differential equations and industry", Math. Sci. 5 $(1980), 1-12$.

[17] J.R. Ockendon, "Linear and nonlinear stability of a class of moving boundary problems", Free boundary problems, 444-478 (Francesco Severi, Rome, 1980).

[18] J.R. Ockendon and W.R. Hodgkins, Moving boundary problems in heat flow and diffusion (Proc. Conf. University of Oxford, March 1974. Clarendon Press, Oxford, 1975).

[19] O.A. OleTnik, "A method of solution of the general Stefan problem", Soviet Math. Dokl. 1 (1960), 1350-1354.

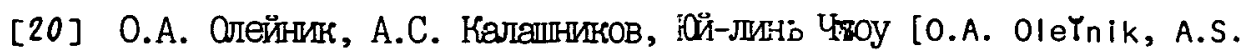
Kalašinkov, YuT-Lin' Č̌ou], "Задаuа Коши и қраевые задачи дия уравнений типа нестационариой филытаци" [The Cauchy problem and boundary problems for equations of the type of non-station stationary filtration], Izv. Akad. Nauk SSSR Ser. Mat. 22 (1958), 667-704.

[21] J.R. Philip, "Flow in porous media", Ann. Rev. Fluid Mech. 2 (1970), $177-204$. 
[22] N. Riley, "Unsteady heat transfer for a flow over a flat plate", $J$. Fluid Mech. 17 (1963), 97-104.

[23] S. Rogers, "Some aspects of Stefan type problems", (D. Phil thesis, University of Oxford, 1977).

[24] L.1. Rubenštein, The Stefan problem (Translations of Mathematical Monographs, 27. American Mathematical Society, Providence, Rhode Island, 1971).

[25] K. Stewartson, "On the impulsive motion of a flat plate in a viscous fluid", Quart. J. Mech. Appl. Math. 4 (1951), 182-198.

[26] K. Stewartson, "On the impulsive motion of a flat plate in a viscous fluid. II", Quart. J. Mech. Appl. Math. 26 (1973), 143-152.

[27] A.B. Tayler, "Mathematical modelling and numerical analysis", Bull. Inst. Math. Appl. 16 (1980), 7-10.

[28] A.B. Tayler, "Mathematical modelling: art or science?", Math. Intelligencer 3 (1981), 66-69.

[29] A.B. Tayler and M.O. Nicholas, "Unsteady slow flows over a cooled flat plate", IMA J. Appl. Math. 28 (1982), 75-91.

[30] D.G. Wilson, Alan D. Solomon, Paul T. Boggs, Moving boundary problems (Academic Press [Harcourt Brace Jovanovich], New York, San Francisco, London, 1978).

Mathematical Institute,

Oxford,

England. 